\title{
Measuring cosmic bulk flows with Type la supernovae from the Nearby Supernova Factory (Corrigendum)
}

U. Feindt ${ }^{1,2}$, M. Kerschhaggl ${ }^{2}$, M. Kowalski ${ }^{1}$, G. Aldering ${ }^{3}$, P. Antilogus ${ }^{4}$, C. Aragon ${ }^{3}$, S. Bailey ${ }^{3}$, C. Baltay ${ }^{5}$, S. Bongard ${ }^{4}$, C. Buton ${ }^{2}$, A. Canto ${ }^{4}$, F. Cellier-Holzem ${ }^{4}$, M. Childress ${ }^{6}$, N. Chotard ${ }^{7,8}$, Y. Copin ${ }^{7,8}$, H. K. Fakhouri ${ }^{3,9}$, E. Gangler ${ }^{7}$, J. Guy ${ }^{4}$, A. Kim ${ }^{3}$, P. Nugent ${ }^{10,11}$, J. Nordin ${ }^{1}$, K. Paech ${ }^{2}$, R. Pain ${ }^{4}$, E. Pecontal ${ }^{12}$, R. Pereira ${ }^{7}$, S. Perlmutter ${ }^{3,8}$, D. Rabinowitz ${ }^{5}$, M. Rigault ${ }^{1}$, K. Runge ${ }^{3}$, C. Saunders ${ }^{3}$, R. Scalzo ${ }^{6}$, G. Smadja ${ }^{7}$, C. Tao ${ }^{13,14}$, R. C. Thomas ${ }^{9}$, B. A. Weaver ${ }^{15}$, and C. $\mathrm{Wu}^{4,16}$

\footnotetext{
${ }^{1}$ Institut für Physik, Humboldt-Universität zu Berlin, Newtonstraße 15, 12489 Berlin, Germany e-mail: feindt@physik.hu-berlin.de

2 Physikalisches Institut, Universität Bonn, Nußallee 12, 53115 Bonn, Germany e-mail: mkersch@physik. uni-bonn. de

3 Physics Division, Lawrence Berkeley National Laboratory, 1 Cyclotron Road, Berkeley, CA 94720, USA

${ }^{4}$ Laboratoire de Physique Nucléaire et des Hautes Énergies, Université Pierre et Marie Curie Paris 6, Université Paris Diderot Paris 7, CNRS-IN2P3, 4 place Jussieu, 75252 Paris Cedex 05, France

5 Department of Physics, Yale University, New Haven, CT 06250-8121, USA

${ }^{6}$ Research School of Astronomy and Astrophysics, Australian National University, Canberra, ACT 2611, Australia

7 Université de Lyon, 69622 Lyon, France

8 Université de Lyon 1, Villeurbanne, CNRS/IN2P3, Institut de Physique Nucléaire de Lyon, 69622 Lyon, France

9 Department of Physics, University of California Berkeley, 366 LeConte Hall MC 7300, Berkeley, CA 94720-7300, USA

${ }^{10}$ Computational Cosmology Center, Computational Research Division, Lawrence Berkeley National Laboratory, 1 Cyclotron Road MS 50B-4206, Berkeley, CA 94720, USA

11 Department of Astronomy, B-20 Hearst Field Annex \# 3411, University of California, Berkeley, CA 94720-3411, USA

12 Centre de Recherche Astronomique de Lyon, Université Lyon 1, 9 avenue Charles André, 69561 Saint Genis Laval Cedex, France

13 Centre de Physique des Particules de Marseille, 163, avenue de Luminy, Case 902, 13288 Marseille Cedex 09, France

14 Tsinghua Center for Astrophysics, Tsinghua University, 100084 Beijing, PR China

15 Center for Cosmology and Particle Physics, New York University, 4 Washington Place, New York, NY 10003, USA

16 National Astronomical Observatories, Chinese Academy of Sciences, 100012 Beijing, PR China
}

A\&A 560, A90 (2013), DOI: 10.1051/0004-6361/201321880

Key words. cosmology: observations - cosmological parameters - large-scale structure of Universe - supernovae: general errata, addenda

In Table 1 of Feindt et al. (2013), the $p$-values for the dipole fit method were calculated incorrectly. While the $\chi^{2}$-function was fully minimized for the real data, it was evaluated on an inadequately large grid for the simulated data. As there were no errors in the $\chi^{2}$-values of the real data, the best-fit values are unaffected.

The correct $p$-values are listed in the table below. Since we observed fluctuations in the results between runs using 5000 random realizations, we increased the number of realizations to 250000 . The main conclusions of the paper are not affected by the changed numbers, because the main result of this method - that there is no evidence for a backside infall behind the Shapley Supercluster - remains the same. The higher $p$-value in the third redshift bin $(0.045<z<0.06)$ does not contradict this observation.

We would like to thank Jeppe Trøst Nielsen for bringing this error to our attention and confirming the corrected numbers.

\section{References}

Feindt, U., Kerschhaggl, M., Kowalski, M., et al. 2013, A\&A, 560, A90 
Table 1. Reconstructed directions (in Galactic coordinates) and corrected p-values of maximum anisotropy according to a dipole fit in different redshift bins for the Union2 and SNFACTORY datasets and their combination.

\begin{tabular}{|c|c|c|c|c|c|c|}
\hline \multirow[t]{2}{*}{ Union2 } & \multirow[b]{2}{*}{$N_{\mathrm{SN}}$} & \multirow[b]{2}{*}{$z$} & \multicolumn{4}{|c|}{ Dipole fit } \\
\hline & & & $l$ & $b$ & $v_{\mathrm{DF}}\left[\mathrm{km} \mathrm{s}^{-1}\right]$ & $p$-value \\
\hline & 109 & $0.015-0.035$ & $290(22)$ & $15(18)$ & 292(96) & 0.015 \\
\hline & 16 & $0.035-0.045$ & $331(59)$ & $-7(37)$ & $496(468)$ & 0.732 \\
\hline & 17 & $0.045-0.060$ & $39(45)$ & $-36(28)$ & $870(490)$ & 0.154 \\
\hline & 23 & $0.060-0.100$ & $54(93)$ & $-10(53)$ & $509(601)$ & 0.783 \\
\hline & 55 & $0.100-0.200$ & $256(90)$ & $-18(34)$ & $1238(1976)$ & 0.388 \\
\hline & 62 & $0.200-0.300$ & $14(131)$ & 11(75) & $1221(1458)$ & 0.644 \\
\hline & 62 & $0.300-0.400$ & $257(84)$ & $-36(27)$ & $2590(2841)$ & 0.349 \\
\hline & 58 & $0.400-0.500$ & 161(48) & 28(29) & $4190(4014)$ & 0.711 \\
\hline & 44 & $0.500-0.600$ & $15(100)$ & $-17(33)$ & $3977(4113)$ & 0.715 \\
\hline & 50 & $0.600-0.800$ & $343(81)$ & $-50(43)$ & $5576(4279)$ & 0.313 \\
\hline & 60 & $0.800-1.400$ & $75(55)$ & $-14(28)$ & $7238(8933)$ & 0.860 \\
\hline \multicolumn{7}{|c|}{ SNFACTORY } \\
\hline & 20 & $0.015-0.035$ & 104(95) & $26(44)$ & $229(410)$ & 0.896 \\
\hline & 20 & $0.035-0.045$ & $286(70)$ & $-7(42)$ & $484(516)$ & 0.861 \\
\hline & 21 & $0.045-0.060$ & $330(48)$ & $48(46)$ & $941(390)$ & 0.016 \\
\hline & 54 & $0.060-0.100$ & $252(134)$ & $5(75)$ & $232(360)$ & 0.909 \\
\hline \multicolumn{7}{|c|}{ Union2+SNFACTORY } \\
\hline & $128^{*}$ & $0.015-0.035$ & $298(25)$ & $15(20)$ & $243(88)$ & 0.027 \\
\hline & 36 & $0.035-0.045$ & $302(48)$ & $-12(26)$ & $452(314)$ & 0.560 \\
\hline & 38 & $0.045-0.060$ & $359(32)$ & $14(27)$ & $650(398)$ & 0.244 \\
\hline & 77 & $0.060-0.100$ & $285(234)$ & $-23(112)$ & $105(401)$ & 0.991 \\
\hline
\end{tabular}

Notes. The only change relative to Feindt et al. (2013) is the correction of the $p$-values for the dipole fit. ${ }^{(*)}$ SN 2005eu (=SNF20051003-004) is included in both datasets. The Union 2 measurement was used for the combined datasets. 